\title{
EDITORIAL
}

\section{National Tuberculosis Control Programme (NTP): Achieved and Yet to Achieve}

(Birdem Med J 2013; 3(1): 1-3 )

Tuberculosis Global Burden: Tuberculosis (TB) is contagious and airborne. There were 9. 4 million new TB cases in 2009. About 1.7 million people died from TB in 2009 which is equal to 4700 deaths a day. The WHO South-East Asia region has an estimated 5 million prevalent and 3. 3 million incident cases of tuberculosis annually. WHO declared TB a global emergency in1993 and recommended the Directly Observed treatment, Short course (DOTS) strategy. WHO ranks Bangladesh $6^{\text {th }}$ among the world's 22high burden TB countries.

Bangladesh Scenario: Tuberculosis is a major public health problem in Bangladesh. Under the mycobacterial disease control (MBDC) unit of Directorate General of Health Services (DGHS), national tuberculosis control programme (NTP) is working with a mission of eliminating TB from Bangladesh. It's main short-term objectives were to sustain the global targets of achieving at least $70 \%$ case detection and $85 \%$ treatment success among new smear positive case under DOTS. The medium term objectives included reaching the TB related millennium development goals. The NTP adopted the DOTS strategy in November 1993. By 2007 the DOTS services were made available throughout the country including metropolitan cities. The NTP has achieved $85 \%$ treatment success since 2003 . The programme has successfully treated almost $92 \%$ of the new smear positive cases registered in 2009 and has detected 70 . $5 \%$ of the estimated new smear positive cases in 2010(assuming that new smear positive cases is 100/ 100000 cases).

Evolution of TB control programme: In 1965 tuberculosis services were mainly curative and based in TB clinics and TB hospitals. TB services were expanded to 124 upazilla health complexes during the $2^{\text {nd }}$ health and population plan(1980-86) and were operationally integrated with leprosy during the $3^{\text {rd }}$ health and population plan(1986-91) under the mycobacterial disease control( MBDC) unit of the directorate general of health services(DGHS). Since adoption of DOTS strategy the NTP has achieved tremendous success. The country in fact is implementing stop TB strategy since 2006.

\section{ESTIMATED TB burden in Bangladesh:}

Mortality rate (excluding HIV) :43/100 000. Prevalence rate (all cases including HIV) :411/100 000.

Incidence rate (all cases including HIV) : 225/100 000. Incidence rate (all cases including HIV) :225/100 000. Incidence rate (all cases including HIV) :225/100 000. . Incidence rate (HIV positive TB cases) :0. 45/100 000. Proportion of new TB cases with MDR -TB : 2. 1\%. Proportion re-treatment TB cases with MDR-TB :28\%

The places where free of charge diagnostic and treatment services for TB are available:

1. All upazilla health complexes . 2. 44 chest disease clinics. 3. chest disease hospitals(previous segregation hospitals) linked to the chest disease clinics4. 4 divisional chest disease hospitals 5 . The National Institute of Diseases of the Chest and Hospital(NIDCH) Dhaka 6. The Government leprosy hospital Nilphamari 7. District Sadar Hospitals 8. Urban health centers in all metropolitan cities (Go B and NGOs) 9. Public and private medical college hospitals. 10. Work places11. Prisons . 12. Combined Military Hospitals and other defense hospitals

Stop TB Strategy at a glance: The vision is to have a TB free world with a goal to dramatically reduce the global burden of TB by 2015. It's objectives are to achieve universal access to high quality care for all people with TB and protect vulnerable populations from TB,TB/HIV and drug resistance TB. It's target is to reduce the prevalence and death due to TB by $50 \%$ compared with a baseline of 1990 and to eliminate TB as a public health problem by 2050 .

Treatment Success: Nationwide Treatment success rates under DOTS have been consistently high from 
the beginning and crossed the global target of $85 \%$ in 2003. After strengthening DOTS and ACMS (advocacy, communication and social mobilization) activities,unfavorable outcomes have been remarkably reduced. As a result this treatment success rate has improved further to reach89\% for the cases registered in 2004. The NTP has been maintaining over 91\% success rate since 2005. In fact NTP has successfully treated 100254(92\%) of the109012 new smear positive cases registered in 2009. The default rate was $1.75 \%$ while $3.7 \%$ of the patients have died during treatment.

Treatment outcome of relapse, new smear-negative and extrapulmonary (new) cases: In 2009 a total of 3920 relapse,25009 smear-negative and 22017 extrapulmonary TB case were registered. The treatment success rate of relapse cases was $81.86 \%$ and treatment completion rates of smear negative and extra- pulmonary cases were 88. 72\% and 88. 93\% respectively. During the course of treatment210(5. 365\% )relapse,1202(4. 81\%)smear negative and 705(3. 20\%)extra pulmonary cases had died and overall death rate was 4. $26 \%$.

Laboratories: Sputum microscopy under NTP was performed in1050 laboratories across the country. All 1050 laboratories were brought under the quality assurance network of the EQA centres. Assessment reports had been received from these EQA centers. A national tuberculosis reference laboratory(NTRL) formally started functioning on $27^{\text {th }}$ June 2007 . It is the only national level laboratory GLC (green light committee)approved project. NTRL is the WHO/The union recommended TB reference laboratory of NTP. In December 2010NTP has started the first national drug resistance survey in collaboration with NTRL. Regional tuberculosis reference Laboratory (RTRL) was inaugurated in Rajshahi on May 2008 and in Chittagong in October 2010, while the other one in Khulna is in progress. In 2010 AFB microscopy was done in18222, with 1912 positive cases. 3620 cultures were done while sensitivity was done in 402 and total MDR detected in the year was 261. In 2010 MDRTB cases treated in chest diseases hospital were 173 .

Quality Assurance: Quality assurance of TB control programme is carried out in two ways: internal quality assurance by a QA team and external quality assessment (EQA) of microscopy centers by EQA laboratory. Internal quality control is performed by a supervisory visit and feedback to local, regional and central level authorities.
A standardized checklist has been developed and utilized by the NTP and BRAC. It's activities include rechecking of positive and negative slides, verifying records and reports, observing DOTs, patient follow up, examining quality of smears and staining, overseeing supply and storage of drugs and reagents, observing laboratory facilities and revewing laboratory and treatment data. Up to June 2010,in collaboration with NTP,BRAC and other NGOs, a total of 37 EQA centres were established throughout the country. Most of EQA centers are established at the Chest disease clinics under the close supervision of junior consultants who are called EQA coordinators and who play a major role for blinded rechecking system. District level supervisors and medical officers at upazilla health complexes collect five slides from each diagnostic /microscopy centers randomly every month. The slides and report are then sent to the EQA coordinator for rechecking. Regular feedback is provided from EQA to microscopy center after assessment of these slides for improving quality of smearing and staining. If any slide is discordent at the first controller(EQA coordinator) level the slide is sent to the second controller for further rechecking at central level. The first controller also visits the microscopy centre for assessing the quality of smearing, staining and also the quality of reagent and the condition of microscopy.

Training Courses and meeting: NTP is being primarily responsible for training, planning all aspects of training and workshop with government and non-government entities to determine training content, develop training materials, identify health staff to be trained, ensure training course implementation and follow up for new hires and maintenance of training. A total of 272 health managers, medical doctors, health workers and volunteers and 981 'shasthyashebika' were trained. Orientation of 22909 village doctors and 2014 pharmacists were conducted and 3830 HIV/AIDS NGO workers were oriented on TB up to June 2011.

ACMS (Advocacy, communication and social mobilization activities):Were conducted throught the country. Orientation, round table meetings and talk shows were arranged. It was done through electronic medias (7 TV spots were aired on 7 channels, a total of 3874 times and six radio spots were aired on 4 radio channels in a total of 7068 times)and print media (51 articles and 422 reports were published in newspapers). 
1576 social mobilization events of folk songs and popular theatre were conducted upto June 2011. Business organizations(viz. BGMEA-Bangladesh Garment Manufacture and Exporters Association)) social organizations(viz NATAB-national anti tuberculosis association of Bangladesh), politicians, celebrities and many others contributed in different ways.

Fund: The global fund to fight AIDS,TB and malaria(GFATM):Since it's creation in 2002, the global fund has become the main financer of programmes to fight AIDS,TB and malaria with approved funding of US\$21. 7 billion for more than 600 programmes in 150 countries(as of December 2010). These funds are flown through approved principal recipient(PR);PR-economic relations division(ERD), Government of Bangladesh and PR-BRAC

Partnership: the national TB control programme under the directorate general of health services(DGHS) has been implementing TB control strategies in collaboration with the NGOs. Specific areas are assigned to NGOs through existing and/or new memorandum of understanding(MOU) for the implementation of programmes. The ministry of health and family welfare (MoH\& FW)is responsible for programme coordination, management, national guidelines for treatment and LABORATORY services, guideline for human resource development(HRD),strategies for ACSM, training of programme coordinators, supervisors and laboratory staffs, procurement and distribution of drugs and laboratory supplies, monitoring and evaluation. Since 2004 BRAC has been serving as one of the principal recipients for TB in Bangladesh. Currently there are 43 NGOs participating as sub -recipients in the national programme under BRAC. The PR-NGO steering committee comprised of representative from PR-BRAC, SRs, NTP and WHO acts as a platform for ensuring the implementation of GFATM fund through partnerships. Well established and effective partnership between government and NGOs helped to increase coverage, case detection and quality of treatment in the past few years. Joint planning and supervision by NTP and partner NGOs helped in identifying gaps in terms of activities and resources, and improving the quality of the programme.

Limitation: Some challenges stillremain. Case detection is low in urban areas and in a few rural areas. Proportion of extra pulmonary, smear negative and child TB cases among the total cases are low. One promising strategy to address these weaknesses is the intensification of collaboration with relevant public and corporate sectors, hospitals and private health care providers. Drug resistance has been identified as a growing concern. NTP started MDR project at the end of2007. From August 2008 to June 2011 a total of575 cases were enrolled in treatment at NIDCH. DST(drug sensitivity test) service is still limited and only a few number of MDR cases are diagnosed and treated by NTP. Uninterrupted supply of drugs and logistics are crucial for TB control.

\section{Conclusion:}

The achievement of 2011 in addressing TB in Bangladesh reflect the continued implementation, expansion and refinement of an effective strategy enabled by the Global Fund resources and facilitated by a broad range of in-country partners. The continued commitment in Bangladesh at all levels and the mutual spirits of partnership will contribute towards reaching the Millenium Development goals (MDG) set for 2015. 